\title{
Renal infarction in COVID-19 patient
}

\author{
Ahmad Ammous $^{1}$ (D) Madeeha Abdul Ghaffar $^{1} \cdot$ Elie El-Charabaty ${ }^{1} \cdot$ Suzanne El-Sayegh $^{1}$
}

Received: 16 July 2020 / Accepted: 14 September 2020 / Published online: 29 October 2020

(c) Italian Society of Nephrology 2020

Keywords COVID-19 · Renal infarct · Acute kidney injury · Thrombotic complications

\section{Nephrology picture}

A 62 years old male with a history of hypertension and asthma presented to the emergency department after sudden onset of left upper quadrant abdominal pain. The patient had been discharged from the hospital 2 days prior, when he had presented for 10 days duration of shortness of breath and was diagnosed with moderate COVID-19 infection. Patient had required oxygen by nasal cannula for the first 3 days of his hospital stay, and was discharged on the fourth day. During his entire stay he was receiving venous thromboembolism prophylaxis with low molecular weight heparin. Patient denied history of tobacco, alcohol or recreational drug use.

On presentation the patient was hemodynamically stable; his physical exam revealed left upper quadrant abdominal tenderness but was otherwise unremarkable. ECG showed normal sinus rhythm. Lab studies were notable for a D-dimer of $2672 \mathrm{ng} / \mathrm{mL}$, and positive Antiphospholipid antibodies, specifically Anticardiolipin IgM antibodies. Urinalysis was normal. Computerized Tomography of the abdomen and pelvis with intravenous contrast demonstrated left renal infarcts, as well as a thrombus in the left renal arterial branch (Fig. 1). Additional findings of bibasilar opacities consistent with known COVID-19 pneumonia were also noted. The patient was started on anticoagulation with Heparin, then transitioned to a novel oral anticoagulant and discharged home. The patient was contacted 6 weeks after discharge, reported feeling well, denied any complications. Repeat blood work four weeks after discharge showed stable kidney function.
Ahmad Ammous

aammous@northwell.edu

1 Department of Internal Medicine, Staten Island University Hospital, NY, Staten Island, USA

\section{Discussion}

COVID-19 is associated with a hypercoagulable state. There are two proposed mechanism for this phenomena, disseminated intravascular coagulation (DIC) and endothelialopathy. A systemic inflammatory response is generated in response to invading pathogens, generating thrombin, fibrin and other coagulation factors. These act to form thrombi in micro-vessels that will capture pathogens and prevent tissue invasion. DIC occurs when these thrombi are unable to contain the spread of pathogens and damaged cells, resulting in excessive inflammation and clot formation. Endothelialopathy occurs when SARS-CoV-2 binds to blood vessel endothelial cells using their angiotensin-converting enzyme 2 receptor. These viruses then replicate, causing an infiltration of inflammatory cells that induce endothelial cell apoptosis [1].

Various venous and arterial complications of COVID-19 have been described [2]. This is a case of COVID-19 complicated by renal infarct. The patient had no personal or family history of cardiac arrhythmia, rheumatological disease or malignancy. Workup revealed positive antiphosholipid antibodies. These antibodies have been detected with other viral infections, often in cases not complicated by thrombosis [3]. Therefore the clinical significance of this finding is uncertain. The patient had normal kidney function up to four weeks after the event, likely because the infarct only affected a small part of the left kidney. However this does not preclude the hypothesis that thrombosis is a contributing factor to the development of acute kidney injury (AKI) in COVID-19 patients [4, 5]. Further work is needed to study the effect of anticoagulation in prophylactic and therapeutic doses in COVID-19 patients, and its ability to protect against the development of AKI. 

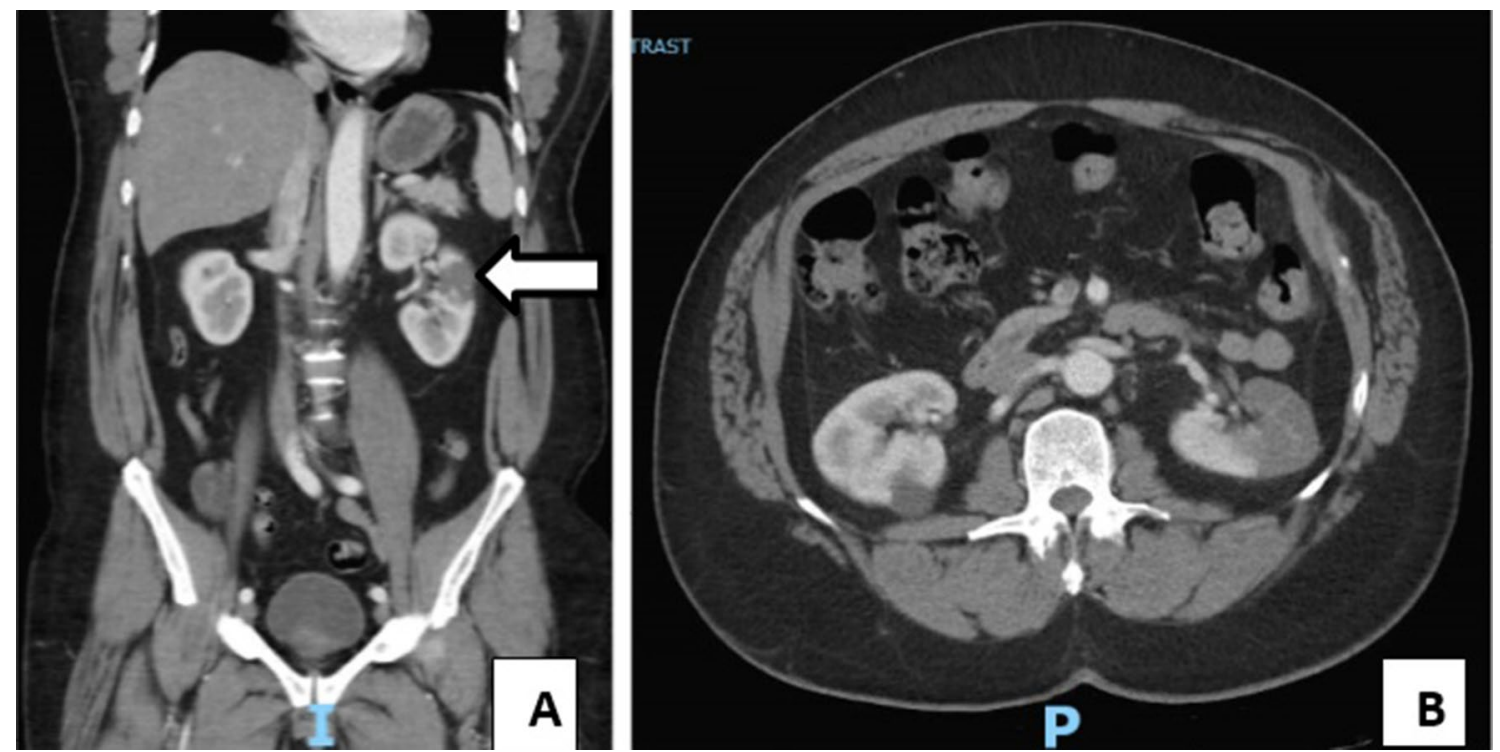

Fig. 1 Computerized tomography (CT) showing: a Wedge shaped area of decreased enhancement in the left kidney (arrow), consistent with renal infarct. b Central filling defect in left renal arterial branch

Acknowledgements We would like to acknowledge the contributions of the Northwell Health COVID-19 Research Consortium. We would also like to acknowledge Jihyn Kang, MD of the Radiology department at Staten Island University hospital, for her help selecting the CT scan images.

The initial characteristics of 5700 COVID-19 patients from Northwell are presented elsewhere. This case series presented in-depth nephrology results not presented in that article.

Richardson S et al (2020) Presenting characteristics, comorbidities, and outcomes among 5700 patients hospitalized with COVID-19 in the New York City area. JAMA.

Funding None.

\section{Compliance with ethical standards}

Conflict of interest The authors declare that they have no relevant financial interests.

Ethics approval This study was approved by Northwell Health Institutional Review Board.

Consent to participate The authors declare that they have obtained from the subject of the study.

\section{References}

1. Connors JM, Levy JH (2020) COVID-19 and its implications for thrombosis and anticoagulation. Blood 135(23):2033-2040

2. Guan WJ, Ni ZY, Hu Y et al (2020) Clinical characteristics of coronavirus disease 2019 in China. N Engl J Med. https://doi. org/10.1056/NEJMoa2002032

3. Uthman IW, Gharavi AE (2002) Viral infections and antiphospholipid antibodies. Semin Arthritis Rheum 31(4):256-263

4. Batlle D, Soler MJ, Sparks MA et al (2020) Acute kidney injury in COVID-19: emerging evidence of a distinct pathophysiology. J Am Soc Nephrol 31(7):1380-1383. https://doi.org/10.1681/ ASN.2020040419

5. Farouk SS, Fiaccadori E, Cravedi $P$ et al (2020) COVID-19 and the kidney: what we think we know so far and what we don't. J Nephrol. https://doi.org/10.1007/s40620-020-00789-y

Publisher's Note Springer Nature remains neutral with regard to jurisdictional claims in published maps and institutional affiliations. 